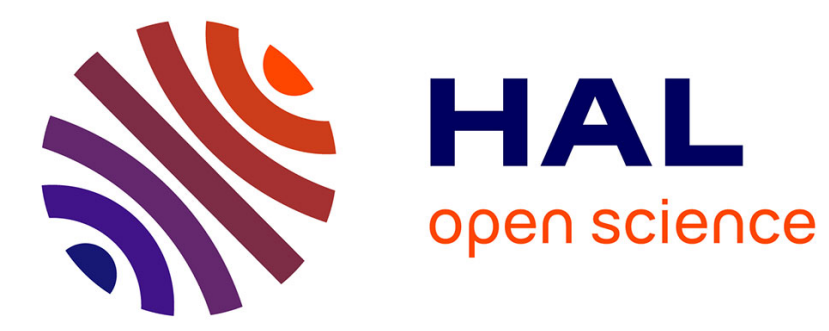

\title{
Les façades institutionnelles: ce que montrent les apparences des institutions
}

Vanessa Codaccioni, Nicolas Maisetti, Florent Pouponneau

\section{To cite this version:}

Vanessa Codaccioni, Nicolas Maisetti, Florent Pouponneau. Les façades institutionnelles: ce que montrent les apparences des institutions. Sociétés contemporaines, 2012. hal-01680690

\section{HAL Id: hal-01680690 \\ https://hal.science/hal-01680690}

Submitted on 11 Jan 2018

HAL is a multi-disciplinary open access archive for the deposit and dissemination of scientific research documents, whether they are published or not. The documents may come from teaching and research institutions in France or abroad, or from public or private research centers.
L'archive ouverte pluridisciplinaire HAL, est destinée au dépôt et à la diffusion de documents scientifiques de niveau recherche, publiés ou non, émanant des établissements d'enseignement et de recherche français ou étrangers, des laboratoires publics ou privés. 


\section{Vanessa CODACCIONI, Nicolas MAISETTI, FlorentPOUPONNEAU}

\section{Les façades institutionnelles : ce que montrent les apparences des institutions}

C

onfrontés aux stratégies discursives et pratiques de rationalisation, de valorisation ou de mise en cohérence des institutions, les chercheurs en sciences sociales peuvent être tentés d'adopter deux attitudes. La première consiste à s'approprier ces discours institutionnels en les promouvant au rang d'explicitation scientifique. La seconde tend à l'inverse à les réduire à des leurres faisant obstacle à l'analyse, et, à ce titre, devant être écartés. Dans les deux cas, le travail de (re)présentation qui fait en partie exister les institutions tend à être négligé. Néanmoins, et c'est le pari des contributions rassemblées dans ce numéro, ces efforts pour paraitre peuvent aussi constituer des objets de recherche pertinents pour mieux appréhender le fonctionnement des institutions.

Sans être spécifiquement nommées comme telles, les « façades » ont fait l'objet de plusieurs travaux révélant les diverses logiques pouvant sous-tendre leur production et leur entretien : accéder au champ politique (Pudal, 1989); se doter de vitrines légales (Crettiez, 2008) ; ou encore donner de l'institution « une image émouvante et digne d'éloges » (Déloye, Haroche, Ihl, 1996). Si ces études soulignent l'enjeu de ces présentations de soi et témoignent de l'investissement dont elles sont l'objet, peu d'entre elles ont spécifiquement porté sur les « apparences » institutionnelles et sur leurs modalités sociales de production et d'existence. C'est ce vide qu'entend contribuer à combler ce dossier à partir d'une réflexion sur la notion de « façade », envisagée comme un « instrument d'objectivation ${ }^{2}$ » utile à l'analyse des institutions et de leurs apparences.

1/ Ce dossier est issu du colloque « Les façades institutionnelles. Constructions, logiques sociales et appropriations des orthodoxies », École doctorale de science politique de l'Université Paris 1, le 25 septembre 2009, co-organisé avec Simon Mas et Sylvain Antichan. Nous remercions ce dernier pour les lectures et les conseils dont a pu bénéficier ce texte.

2/ Comme l'a fait Muriel Darmon à propos de la notion interactionniste de « carrière » (2008). 
Au sens d'Erving Goffman, les «façades» peuvent être entendues comme « la partie de la représentation qui a pour fonction normale d'établir et de fixer la définition de la situation qui est proposée aux observateurs » (1973, p. 29). L'auteur conçoit dans ce cadre deux espaces sociaux connexes, d'un côté la scène, et de l'autre les coulisses, pour montrer comment le comportement des acteurs varie en fonction des lieux possibles et du public à convaincre. En pensant ces deux espaces comme un continuum de pratiques et de discours, l'analyse interactionniste permet en principe de dépasser une série d'oppositions binaires partiellement inopérantes - devant/derrière, vrai/faux, officiel/officieux. Elle invite aussi à penser, intégrée à une sociologie des institutions, les ajustements perpétuels des acteurs et de leurs institutions pour imposer à leurs publics diversifiés une représentation légitime d'eux-mêmes. Il ne s'agit donc pas de «soupçonner le pire et le traquer », comme le dirait Howard Becker (2002), ou d'adopter une posture de dévoilement, mais plutôt d'être attentif aux variations des registres de présentation de soi en fonction des moments et des lieux dans lesquels une définition spécifique de la situation semble devoir s'imposer.

À ce titre, l'apport principal des «façades » telles qu'elles ont été pensées par Goffman est de permettre de saisir la pluralité des registres de présentation de soi, de leurs supports et de leurs modalités de diffusion. Selon la typologie dressée par Goffman, les façades comprendraient d'une part le « décor», un ensemble d'« éléments scéniques » comme le mobilier, la décoration ou la manière dont sont disposés les objets, et, d'autre part, ce qu'il appelle la «façade personnelle» propre à l'acteur mis en scène. C'est par exemple le cas des insignes hiérarchiques, des vêtements ou des accessoires, des gestes, des façons de parler et de dire, de se mouvoir. Et, malgré de possibles dissonances - les « gaffes», les «fausses notes» ou les « ratés » d'imposition de sens - ces éléments d'appareillage symbolique seraient très majoritairement congruents et se renforceraient mutuellement pour donner à voir une présentation de soi uniforme et cohérente (Goffman, 1973).

Néanmoins, si l'usage goffmanien des façades donne la possibilité d'aborder frontalement les « appareillages symboliques » dont se dote un acteur en représentation, il a été pensé pour analyser les interactions face-à-face. Il est donc peu adapté à une sociologie des institutions centrée sur les relations entre l'instituant et l'institué et sur des processus d'institutionnalisation (Lagroye, Offerlé, 2011, 
p. 17) ${ }^{3}$. Plus qu'une simple image, la façade apparaît à la fois comme un facteur d'institutionnalisation et comme le produit de cette dernière.

Au-delà de la diversité de leur objet - une coalition municipale, un parti politique, un couvent religieux, et l'image d'un maire - les articles rassemblés dans ce numéro se donnent un même objectif : penser les « apparences » des institutions. La variété des terrains que regroupe ce dossier est donc, d'abord, révélatrice de la large diffusion des pratiques qu'il interroge. Plus encore, cette diversité permet de rapprocher des comportements institutionnels a priori disparates mais qui, au moins à titre d'hypothèse, partageraient des mécanismes politiques et sociaux similaires et produiraient des effets convergents sur le fonctionnement des institutions. Ces études donnent ainsi à voir les procédés de légitimations internes et externes, de codification et de mise en forme des institutions, leur degré d'emprise ou d'unité, mais aussi leur permanence et leur stabilité tout comme les résistances au processus d'institutionnalisation.

La façade de la coalition municipale de Calais étudiée par Nicolas Bué s'adosse aux entreprises de production d'une image unitaire du gouvernement local. Il montre que la façade, loin de se limiter à un « écran qui cache la réalité » comme le suppose son usage métaphorique, est constitutive de l'institution. En partie déterminé par les normes du champ politique, il montre que l'entretien de la façade coalitionnelle est délégué à des « technologies de présentation institutionnelle» discursives (lissage de discours, convocation de références historiques) et matérielles (contrat de coalition, distribution de l'information, processus décisionnel). En outre, et c'est l'un des points négligés par la perspective goffmanienne, son article retrace les conditions de socialisation des membres de la coalition, montrant ainsi comment les logiques d'apprentissage et d'encadrement constituent un enjeu pour le maintien de l'institution. Toutefois, l'auteur souligne aussi les « incartades hétérodoxes » et les «débordements» qui rythment l'entretien de la façade dont la stabilité n'est qu'apparente, si bien que la présentation d'une institution est toujours traversée et constituée par les luttes et les contestations.

\footnotetext{
3/ Dans les contributions de cet ouvrage collectif, l'institutionnalisation y est analysée comme le produit d'une « rencontre » entre deux composantes : ce qui est institué (règles, formes d'organisation, croyances, connaissances) et ce qui est investi par les individus (engagement, adhésion ou résistance). De cette relation dialectique entre l'institué et l'instituant résulte un accord relativement stable, mais continuellement renégocié et réactualisé sur les manières légitimes d'agir au sein de l'institution.
} 
Dans son article sur la guerre d'Algérie, Vanessa Codaccioni choisit d'étudier la «façade » légaliste du PCF pour re-sociologiser la question du «double langage » communiste et se départir à la fois de la posture dénonciatrice des détracteurs du parti et des déclarations contradictoires de ses membres. Évolutive, dépendante des multiples contextes dans lesquels l'organisation partisane est prise, cette façade est constituée de multiples « mises en garde » publiées par les instances dirigeantes dans les organes de presse communiste. Elle vise alors tout autant à légitimer le Parti comme entreprise politique responsable et capable d'intégrer le champ politique central qu'à délimiter les comportements publics acceptables et inacceptables. Ces stratégies discursives produisent en retour des postures, des manières de militer, de dire ou d'écrire dont le degré de conformité varie en fonction de la position dans la hiérarchie du parti et des causes considérées comme dignes d'être défendues. En ce sens, le recours à la façade est ici envisagé comme un moyen de penser les processus d'homogénéisation partisans tout en se détachant d'une analyse totalisante du PCF. Il offre ainsi la possibilité de penser le degré d'emprise qu'une institution souhaite exercer sur ses membres, mais aussi la manière dont elle tente de tenir dans les contextes de crise par la réimposition d'une orthodoxie et d'une orthopraxie.

Yann Raison du Cleuziou, dans son étude de l'ordre dominicain en France aux lendemains de la deuxième guerre mondiale, appréhende le couvent comme une façade. Trois éléments sont distingués : les décors (forme architecturale, ameublement, agencement de l'espace), les apparences personnelles (état, forme et couleur des vêtements, rasage), mais aussi les manières d'être et les pratiques ritualisées (technique de discipline du corps, prescriptions pratiques à suivre). La façade n'est plus seulement une apparence superficielle de l'institution mais un instrument de savoir et de pouvoir qui participe pleinement à l'institutionnalisation du groupe. Les façades, comme mise en forme des conduites et prise de rôle spécifique, sont des dispositifs institutionnels qui permettent à la fois d'imposer des manières de penser et de rendre visible, et donc de contrôler, les déviances des membres au sein de l'institution. Cet usage de la façade permet ainsi de penser la permanence d'une institution.

En posant la question des conditions de réalisation de l'image favorable d'une équipe municipale, Virginie Anquetin se démarque des postures qui se proposent de décrire la «stratégie de communication » des maires. Le cas strasbourgeois est ici mis au service d'une observation fine des modalités de production des «impressions » imposées par « l'équipe en représentation » réunie autour du premier 
magistrat de la ville. L'auteure invite à saisir la façade mayorale non seulement à partir des rôles politiques remplis par le maire, ses conseillers, ses adjoints, son personnel administratif, ainsi que les membres de l'opposition (interne et externe à la majorité municipale), mais également depuis les stratégies d'enrôlement des journalistes locaux. Elle montre que les impressions valorisées qui en résultent ne sont jamais acquises une fois pour toutes et que la façade est régulièrement remise en cause par une série de phénomènes sociaux et politiques (luttes au sein de la majorité municipale, critiques de l'opposition, prises inégales sur le traitement médiatique et incertitudes des effets de légitimation engendrée par l'action publique). La mise en scène du « bon » maire demeure, en conséquence, intrinsèquement liée au travail de contrôle des impressions soumis à des menaces constantes que font peser les malentendus et les ruptures de loyauté sur la mise en ordre lisse de la façade mayorale.

Comme le montrent les contributions rassemblées dans ce numéro, ce dossier invite à trois prolongements par rapport à la perspective goffmannienne des façades.

Goffman a en effet ouvert des pistes de description des « appareillages symboliques » des institutions et de leurs membres. Penser ces « identités» ou ces «versions» officielles renseigne ainsi sur leur capacité à créer une réalité conforme à leurs attentes, et, peut-être surtout, sur ce qu'ils veulent donner à voir. Dès lors, si l'analyse interactionniste a favorisé l'essor de recherches sur les usages des stigmates (Goffman, 1975), elle peut tout autant, lorsqu'elle invite à observer les «façades », donner à penser les catégories dans lesquelles et sous lesquelles les acteurs veulent se voir ranger. « Légaliste » dans le cas du PCF, « unitaire » pour la coalition municipale calaisienne ; " positive» dans le cas de l'institution mayorale ou «dominicain» dans celui de l'ordre conventuel, toutes les contributions rassemblées dans ce numéro accordent une importance particulière aux autodéfinitions des institutions, aux croyances qu'elles engagent et aux pratiques qui les fondent et qui permettent de les stabiliser.

Néanmoins, un protocole de recherche portant sur les logiques de leur construction et de leur entretien reste à élaborer. Certes, les éléments composant une façade peuvent être saisis dans leur matérialité, à travers l'architecture, la disposition spatiale d'un conseil municipal ou d'un couvent, les articles de presse ou les déclarations publiques de dirigeants, les comportements militants, les documents officiels élaborés par les institutions, ou les pratiques ritualisées. Mais 
le repérage empirique d'une façade ne se limite pas nécessairement à ces manifestations les plus visibles. Non seulement parce qu'elle peut être plurielle, en fonction du personnel mobilisé pour la produire, du public visé et du type de regard porté sur elle (sociologique, politique, etc.), mais surtout parce qu'elle n'est jamais figée. L'une des pistes de recherche empruntée dans ce dossier est dès lors de prendre au sérieux ce caractère évolutif des présentations de soi et de s'attacher aux conditions sociales qui les ont rendues possibles. Il s'agit ainsi, en prolongeant la perspective goffmanienne, de se détacher d'une analyse descriptive et fixiste des « façades » afin d'en proposer une analyse processuelle prenant en compte les «apparences » en train de se faire.

L'article de Nicolas Bué montre comment, à partir de 1971, le discours « unitaire » du gouvernement municipal de Calais et sa matérialisation spatiale dépendent des conditions d'accession au pouvoir et des croyances des premiers élus coalisés. À travers l'observation des positions et des relations entre les membres de la coalition, il souligne que l'édification d'une telle façade répond à deux objectifs distincts mais pourtant complémentaires : une définition de ce qui est en train de se jouer et une prescription de comportements destinée aux élus locaux. L'étude de la genèse de la formation du gouvernement de coalition permet ainsi de dégager comment une «façade » est à la fois dépendante des conditions historiques dans lesquelles elle s'impose.

Dans cette perspective, l'article de Vanessa Codaccioni souligne la nécessité de recourir à une analyse processuelle du travail de mise en forme d'une institution. Son observation de la façade légaliste adoptée par le Parti communiste témoigne, malgré sa «fixité » pour les contemporains de la guerre d'Algérie, des facteurs évolutifs l'évolution du conflit armé, sa position dans le champ politique, le degré de répression, les résistances de certains intellectuels ou militants - qui constituent progressivement l'ensemble des pratiques et des discours autorisés à des fins de bonne présentation de soi.

C'est dire aussi que s'intéresser aux apparences des institutions permet d'observer tout ce qui peut faire évoluer, dans un processus continu, les rapports de force institutionnels.

À ce titre, l'un des apports de l'approche en termes de « façades » institutionnelles, à condition de prolonger l'analyse goffmanienne, est d'insister sur leurs modes d'appropriation et sur le rôle qu'y jouent dans leurs interactions les différents acteurs, en fonction de leur 
trajectoire, de leurs positions, de leurs ressources et de leurs « raisons » respectives, entendues à la fois comme intérêts et justifications. Comment une représentation légitime parvient-elle à s'imposer et à acquérir la force de l'évidence au sein d'une institution? Quels acteurs - entrepreneurs en représentation, personnels de renfort ou équipes - sont stratégiques dans ces entreprises? Les observateurs - et lesquels - adhèrent-ils à ces stratégies de présentation?

Entrent dans ce type de questionnement à la fois le problème des «publics» auxquels sont destinées les façades - comme le montre l'article de Virgine Anquetin, ils peuvent être extérieurs aux institutions (journalistes, élus de l'opposition, destinataires de l'action publique et potentiels électeurs) ou parties prenantes (membres de la majorité et de l'administration municipale) - et celui de leur réception par ces mêmes acteurs. Une telle perspective invite alors à observer les effets de ces pratiques sur les croyances des individus. Celles-ci, fonction de propriétés sociales, dépendent alors de mécanismes d'apprentissage ou de socialisation, tout comme d'éventuels « rites d'institution » (Bourdieu, 1982), qui façonnent l'individu soucieux de se conformer aux rôles attendus par l'institution.

Sur ce point, Yann Raison du Cleuziou souligne les moments au cours desquels la façade conventuelle se fait pratiques ritualisées et s'insère dans une logique plus vaste de surveillance des corps et des esprits. La vie monacale rythmée par ses prières, ses tâches, son système de punitions-récompenses et ses repas objectivent un dispositif de vérité et, partant, de la présence divine. En d'autres termes, il montre que la façade n'est pas un trompe-l'œil, mais bien au contraire un espace social panoptique dans lequel chacun des membres de l'institution est tour à tour objet de l'observation et observateurs. L'analyse offre finalement un aperçu détaillé de la manière dont ce que l'auteur appelle une «technologie d'institutionnalisation » parvient à susciter la docilité des membres.

Le cas de la façade mayorale strasbourgeoise étudiée par Virginie Anquetin illustre une dimension complémentaire de la façade en tant qu'entreprise d'encadrement. Elle détaille en effet les conditions d'enrôlement d'une série d'acteurs et de groupes sociaux. Si elle souligne le rôle central joué par le maire dans la mise en cohérence des impressions et dans la distribution des ressources de pouvoir au sein de sa majorité, c'est pour mieux souligner qu'il n'est pas seul engagé dans la fabrication de la façade. Davantage qu'à une description des équipiers, l'enquête conduit à dénouer le fil des interactions entre les journalistes et l'équipe municipale, d'une part, qui ajustent le 
cadrage médiatique, et entre les membres de la majorité, d'autre part, pour lesquels la carrière dépend en partie du maintien de la façade et donc de la manifestation de leur loyauté à l'égard du maire.

L'étude des «façades» incite, comme on le voit, à analyser ensemble, de manière relationnelle, leur réception par les membres des institutions, et le travail entrepris par les entrepreneurs en représentation. L'étude des logiques de réception telle qu'elle est entreprise dans ce dossier interroge ainsi le rapport au rôle des individus, leur capacité variable à le «tenir » ou à le rejeter dans des situations diverses ou face à des épreuves (Lagroye, 1997 et 2002).

Et en effet, s'intéresser aux « apparences » des institutions, c'est aussi rendre compte des failles, des refus et des ruptures de définition qui se produisent au sein des groupes mobilisés. Le troisième prolongement emprunté par les auteur.e.s conduit ainsi à relativiser la prédilection de Goffman pour la congruence des façades. Ils et elles montrent tout l'intérêt de focaliser sur les tensions en amont des présentations de soi collectives, afin de dévoiler les logiques conflictuelles de fabrication de l'apparence. Car, les acteurs ne jouent pas toujours un jeu conforme aux attentes produites par l'institution: ils s'écartent du rôle ou des rôles exigés d'elle et tentent d'en modifier les normes, les règles, les pratiques, parfois même l'orthodoxie.

Le questionnement doit donc porter à la fois sur la manière dont l'institution tient et parvient à produire des comportements adaptés aux façades prédéfinies mais aussi sur les réactions de ses membres à cette emprise sur leur manière d'être, de faire ou de penser. Les problématiques liées à l'obéissance et à la déviance institutionnelles, dont Delphine Dulong a récemment montré toute la complexité en insistant sur la variation de leur perception en fonction des contextes et sur l'aspect stratégique qu'elle peut revêtir pour les «déviants» (2011), sont donc au cœur de ce jeu d'interactions entre l'institution et l'institué.

Dans son article, Nicolas Bué propose ainsi d'analyser les interactions d'acteurs qui ont construit, stabilisé, puis contesté l'orthodoxie de la coalition municipale calaisienne. L'observation des luttes concurrentielles entre ceux qui ont eu intérêt à l'entretien de la façade et ceux qui ont visé à la dévoyer permet de rendre compte des constantes tensions qui ont présidé non seulement au maintien, mais également à la stabilisation de cette façade coalitionnelle. En l'espèce, l'impératif de présentation unitaire n'imprègne pas les discours de 
tous les acteurs, dans la mesure où le maintien de la façade, c'està-dire l'unité du gouvernement municipal, est l'un des enjeux de la compétition politique.

De manière proche, le cas des membres du PCF qui résistent au légalisme et à l'abandon de la radicalité militante (les étudiants, les ouvriers algériens, les intellectuels anticolonialistes) témoigne du jeu permanent des réajustements institutionnels du groupe dirigeant pour faire tenir la façade communiste. Dans un contexte de crise de l'institution, les luttes pour la maintenir ou la dévoyer prennent alors la forme de conflits pour l'obtention ou la conservation des positions de pouvoir en son sein, notamment à l'intérieur du groupe dirigeant où s'opposent ceux qui détiennent le monopole partisan et ceux qui veulent redéfinir l'institution et la diriger. En ce sens, la déstabilisation de la façade du PCF révèle la fragilisation de la domination charismatique de ses dirigeants et leur difficulté à disciplinariser les militants.

Le texte de Yann Raison du Cleuziou prolonge ces réflexions en identifiant dans ces désajustements des moments de fragilisation des façades et de leurs supports. L'observation du couvent dominicain souligne que les phénomènes de saturation des façades institutionnelles n'interviennent pas seulement lorsqu'ils ne suscitent plus l'adhésion. Par l'action d'entrepreneurs de cause, il se substitue à la façade dominicaine de la vie monacale et contemplative, celle d'un Dieu « engagé » dans le monde et les luttes politiques et sociales.

La contribution de Virginie Anquetin, enfin, évoque sur ce point les contraintes qui pèsent sur les processus de socialisation à l'entretien de la façade. De ce fait, les journalistes, les élus de la majorité ou les membres de l'administration municipale ne coopèrent pas tous et avec la même intensité à la bonne présentation du maire. Engagés dans des intérêts professionnels qui ne coïncident pas toujours avec celle-ci, ils peuvent en contester les fondements. En retour, la critique suscite un continu travail de redéfinition des impressions comme le montrent les conflits autour de l'identité « gauche moderne » ou la mise en scène des «politiques municipales ». Ses conclusions rejoignent celles des autres contributions qui témoignent de l'inscription des négociations entourant l'élaboration des façades dans les processus de réinvention permanente des institutions.

Ainsi, non seulement les façades se transforment au gré des modifications contextuelles et de l'investissement de leurs membres, mais elles sont aussi l'objet de conflits qui revêtent la forme de luttes pour les positions de pouvoir dans l'institution. Tout au moins, c'est dans 
l'interaction constante entre différents acteurs aux intérêts complémentaires ou divergents que peuvent être pensées les apparences des institutions et leurs modalités d'existence. Les partenaires retors de la coalition municipale calaisienne, les militants communistes hostiles à l'abandon d'un anticolonialisme plus radical, les conseillers ou les adjoints du maire de Strasbourg déclassés, ou les moines dominicains qui aménagent le respect de la Règle conventuelle sont engagés, selon des modalités spécifiques, dans des luttes visant à se repositionner au sein de l'institution. Marginalisés ou vaincus dans la compétition pour la définition de son apparence, ils se démarquent, par leurs pratiques ou leurs critiques, de la ligne de conduite officielle. Et si elles ont peu d'impact sur l'orthodoxie imposée, et qu'elles se traduisent plutôt par des rappels à l'ordre institutionnels ou par des sorties (volontaires ou non) du groupe, ces stratégies de rupture suggèrent en interne et en externe qu'une autre institution est possible.

Ce dossier révèle ainsi les modes très variés de construction, d'entretien ou d'effacement des (re)présentations que les institutions donnent d'elles-mêmes. Rarement stabilisés, les registres de légitimation des institutions apparaissent comme le produit d'ajustements issus de luttes ou de négociations autour de la définition même des institutions, des rôles attendus, des discours et des pratiques possibles, pensables et acceptables. En ce sens, l'étude des conditions sociales de ces mises en scène d'institution constitue bien un moyen de mieux comprendre le processus d'autonomisation et d'emprise des institutions et « ce qui les fait tenir, durer et s'imposer »(Gaiti, 2006).

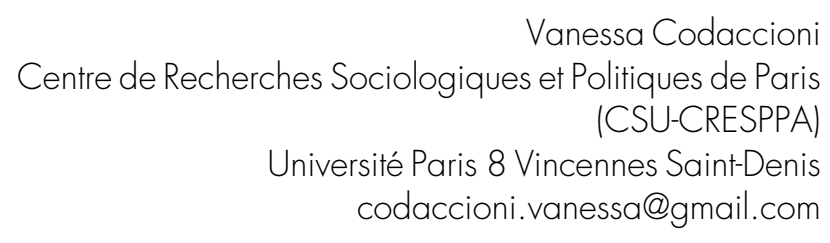
Nicolas Maisetti
Croyances, Histoire, Espaces, Régulation politique
et administrative (CHERPA)
Sciences Po Aix-en-Provence
Nicolas.Maisetti@yahoo.fr

Florent Pouponneau Centre européen de sociologie et de science politique (CRPS-CESSP) Université Paris 1 Panthéon-Sorbonne f.pouponneau@gmail.com 


\section{RÉFÉRENCES BIBLIOGRAPHIQUES}

BECKER H., 2002

BOURDIEU P., 1982

CRETTIEZ X., 2008

DARMON M., 2008

DULONG D., 2011

GAITI B., 2006

GOFFMAN E., 1973

GOFFMAN E., 1977

LAGROYE J., 1997

LAGROYE J., 2002

LAGROYE J., OFFERLÉ M., 2011

PUDAL B., 1989

DÉLOYE Y., HAROCHE Y., IHL O., 1996
Les Ficelles du métier : comment conduire sa recherche en sciences sociales, Paris : La Découverte, coll. Repères.

«Les rites comme actes d'institution », Actes de la recherche en science sociale, vol. $43, \mathrm{n}^{\circ} 43$, p. 58-63.

Les Formes de la violence, Paris : La Découverte, coll. Repères.

« La notion de carrière : un instrument inter-actionniste d'objectivation », Politix, nº 82, p. 149-167.

«Au dedans et en dehors : la subversion en pratique », in : Lagroye J. et Offerlé M. (dir.), Sociologie de l'institution, Paris : Belin, p. 249-265.

«Entre les faits et les choses, la double face de la sociologie politique des institutions », in : Cohen A., Lacroix B., et Riutort P. (dir.), Les Formes de l'activité politique, Paris : PUF, p. 39-64.

La Mise en scène de la vie quotidienne - 1. La présentation de soi, Paris : Minuit.

Stigmates. Les usages sociaux des handicaps, Paris : Minuit.

«On ne subit pas son rôle », entretien préparé et recueilli par Brigitte Gaïti et Frédéric Sawicki, Politix, n 38, p. 7-17.

"L'institution en pratiques », Revue suisse de science politique, vol. $8, n^{\circ} 3 / 4$, p. 114-128.

Sociologie des institutions, Paris : Belin.

Prendre parti. Pour une sociologie historique du PCF, Paris : Presses de sciences Po.

Le Protocole ou la mise en forme de l'ordre politique, Paris : L'Harmattan. 
